\title{
Maximum Interval of Stability and Convergence of Solution of a Forced Mathieu's Equation
}

\author{
Everestus Obinwanne Eze ${ }^{1 *}$, Uchenna Emmanuel Obasi, \\ Rosary Ngozi Ujumadu², Grace Ihuoma Kalu ${ }^{1}$ \\ ${ }^{1}$ Department of Mathematics, Michael Okpara University of Agriculture, Umuahia, Nigeria \\ ${ }^{2}$ Department of Mathematics, Chukwuemeka Odimegwu Ojukwu University, Uli, Nigeria \\ Email: *obinwanneeze@gmail.com, sirurchobasi@gmail.com
}

How to cite this paper: Eze, E.O., Obasi, U.E., Ujumadu, R.N. and Kalu, G.I. (2020) Maximum Interval of Stability and Convergence of Solution of a Forced Mathieu's Equation. World Journal of Mechanics, $\mathbf{1 0}$, 210-219.

https://doi.org/10.4236/wjm.2020.1011015

Received: November 5, 2020

Accepted: November 17, 2020

Published: November 20, 2020

Copyright $\odot 2020$ by author(s) and Scientific Research Publishing Inc. This work is licensed under the Creative Commons Attribution International License (CC BY 4.0).

http://creativecommons.org/licenses/by/4.0/

\begin{abstract}
This paper investigates the maximum interval of stability and convergence of solution of a forced Mathieu's equation, using a combination of Frobenius method and Eigenvalue approach. The results indicated that the equilibrium point was found to be unstable and maximum bounds were found on the derivative of the restoring force showing sharp condition for the existence of periodic solution. Furthermore, the solution to Mathieu's equation converges which extends and improves some results in literature.
\end{abstract}

\section{Keywords}

Frobenius Method, Eigenvalue Approach, Stability, Mathieu's Equation

\section{Introduction}

Consider a harmonically forced Mathieu's equation defined by

$$
\frac{\mathrm{d}^{2} x}{\mathrm{~d} t^{2}}+(w+\varepsilon \cos t) x=f \cos \lambda t
$$

For small $|\varepsilon|$, this equation describes a simple harmonic oscillator whose frequency is a periodic function of time with the boundary condition as;

$$
\begin{aligned}
& x(0)=x(2 \pi) \\
& \dot{x}(0)=\dot{x}(2 \pi)
\end{aligned}
$$

where $\frac{\mathrm{d}^{2} x}{\mathrm{~d} t^{2}}$ is the second derivative with respect to time, $f$ is the amplitude of a periodic driving force, $w$ and $\varepsilon$ are the Mathieu's parameters and $\lambda$ is the angular frequency of the periodic driving force. 
Mathieu's equation is a special case of a linear second order homogenous equation [1]. In [2], Equation (1) was discussed in connection with problem of vibrations in elliptical membrane and developed the leading terms of the series known as Mathieu's function. Mathieu's function was further investigated by a number of researchers who found a considerable amount of results. [3] [4] [5] [6] wrote that Mathieu's differential equation occurs in two main categories involving elliptical geometrics, such as analysis of vibrating modes, elliptical membrane, the propagation modes of elliptic pipes and the oscillation of water in a lake of elliptic shape. Mathieu's equation arises after separating the wave equation using elliptic coordinates. Secondly, problems involving periodic motion are the trajectory of an electron in a periodic array of atoms [7].

Stability is an important concept in linear and nonlinear analysis. For instance, roughly speaking, a physical system is stable if small changes at sometimes cause only a small change in the behavior of the system in future [8]. Analytically, stability is determined by the interval placed on the total derivative of the system form by the given differential equation. Such interval is restricted only on the equilibrium point of the system. Other intervals of interest which have been studied by others researchers in these recent times can be seen in [9] [10]. For linear system, stability and boundedness are equivalent; thus, the search for convergence of solution and maximum interval of stability becomes an issue. Emphasis on stability and convergence has been discussed by many authors. For instance see [11] [12] [13] [14] [15] and their references therein. For other researchers who have worked on stability of non-linear systems, see [16] [17] [18] [19].

Motivated by the above literature and ongoing research in this direction, the objectives of this paper are to investigate the maximum interval of stability and convergence of solution of forced Mathieu's equation. We further prove that the solution converges in that interval of interest and that if all solutions are bounded, then the corresponding point in the $w$ and $\varepsilon$ parameter plane is said to be stable.

\section{Preliminaries}

Definition 2.1. Frobenius method which was named after Ferdinard Geog Frobenius is a method for finding an infinite series solution for a second-order ordinary differential equation of the form;

$$
x^{2} y^{\prime \prime}+p(x) y^{\prime}+q(x) y=0
$$

with $y^{\prime}=\frac{\mathrm{d} y}{\mathrm{~d} x}$ and $y^{\prime \prime}=\frac{\mathrm{d}^{2} y}{\mathrm{~d} x^{2}}$

In the vicinity of the regular singular point $x=0$, we can divide (3) by $x^{2}$ to obtain a differential equation of the form;

$$
y^{\prime \prime}+\frac{p(x)}{x^{2}} y^{\prime}+\frac{q(x)}{x^{2}} y=0
$$


which will not be solvable with regular power series method if either $\frac{p(x)}{x^{2}}$ or $\frac{q(x)}{x^{2}}$ are not analytic at $x=0$. The Frobenius method enables us to make use of powers series solution to solve a different equation, given that $p(x)$ and $q(x)$ are themselves analytic at 0 or being analytic elsewhere. In this case both limit exist at 0 and are finite [20].

Definition 2.2. Stability is a qualitative property of behavior of the solutions of differential equations by which given a reference solution $x^{*}\left(t, t_{0}^{*}, x_{0}^{*}\right)$ of $\dot{x}=x\left(t, t_{0}^{*}, x_{0}^{*}\right) \in \mathbb{R}^{n}, t \geq t_{0}^{*}, t_{0}^{*} \geq 0$, any other solution $x\left(t, t_{0}, x_{0}\right)$ starting close to $x^{*}\left(t, t_{0}^{*}, x_{0}^{*}\right)$ i.e. $t_{0}^{*} \sim t_{0}$ and $x_{0}^{*} \sim x_{0}$ remains close to $x^{*}\left(t, t_{0}^{*}, x_{0}^{*}\right)$ for later times.

\section{Theorem 2.3. Suppose}

(i) $x_{0}$ is a regular singular point and

(ii) $\left(x_{0}-x\right) p(x)$ and $\left(x_{0}-x\right)^{2} q(x)$ are analytic at $x_{0}$

Then Frobenius method is effective at regular singular point of the form.

$$
\left(x-x_{0}\right) \sum_{n=0}^{\infty} a_{n}\left(x-x_{0}\right)^{n}
$$

Theorem 2.4. Assume that $x_{0}=0$ otherwise the point $x=x_{0}$ is a singular point of

$$
P(x) y^{\prime \prime}(x)+Q(x) y^{\prime}+R(x) y(x)=0
$$

and that $Q_{1}(x)=\frac{x Q(x)}{P(x)}$ and $Q_{2}(x)=\frac{x^{2} R(X)}{P(x)}$ are analytic at $x=0$, then they will have Maclaurin series expansion

$$
f(x)=f(0) x+\frac{f^{\prime \prime}(0)}{2 !}+\frac{f^{3}(0)}{3 !} x^{3}+\cdots+\frac{f^{n}(0)}{n !} x^{n}+\cdots
$$

with radius of convergence $r_{1}>0$ and $r_{2}>0$ respectively. That is $Q_{1}(x)=\frac{x Q(x)}{P(x)}=\sum_{n=0}^{\infty} P_{n} X^{n} \quad$ which converges for $|X|<r_{2}$. Then the point $x_{0}=0$ is called a regular singular point of (5).

Theorem 2.5. Consider a power series

$$
f(x)=\sum_{n=0}^{\infty} a_{n} x^{n}
$$

with radius of convergence $R$, then term by term differential and integration of the power series is permitted and does not change the radius of convergence that is;

$$
\begin{gathered}
\frac{\mathrm{d} f}{\mathrm{~d} x}=\frac{\mathrm{d}}{\mathrm{d} x} \sum_{n=0}^{\infty} a_{n} x_{0}=\sum_{n=0}^{\infty} \frac{\mathrm{d}}{\mathrm{d} x} a_{n} x^{n}=\sum_{n=1}^{\infty} n a_{n} x^{n-1},|x|<R \\
\int f(x) \mathrm{d} x=\int \mathrm{d} x \sum_{n=0}^{\infty} a_{n} x^{n}=\sum_{n=0}^{\infty} \int \mathrm{d} x a_{n} x^{n}=\sum_{n=0}^{\infty} \frac{a_{n} x^{n+1}}{n+1},|x|<R
\end{gathered}
$$

Theorem 2.6. Let $A$ be an $n * n$ matrix and let the eigenvalue of $A$ be de- 
noted by $\lambda(A)$ and consider the linear system of differential equation

$$
\dot{x}(t)=A x(t), x \in R^{n}
$$

(i) If $\operatorname{Re}(\lambda(A)) \leq 0$ and all the eigenvalues of $A$ with real part zero are simple, then zero is a stable fixed point of $(10)$

(ii) If $\operatorname{Re} \lambda(A)<0$, then zero is a globally asymptotically stable solution of (10)

(iii) If there is an eigenvalue of $A$ with positive real part, then zero is unstable

\section{Results and Discussion}

\subsection{Stability Analysis of Mathieu Equation}

We consider the equation

$$
\frac{\mathrm{d}^{2} x}{\mathrm{~d} t^{2}}+p x=0
$$

where $p$ is a positive constant. Equation (11) can be written as

$$
\ddot{x}+p x=0
$$

Let $x_{1}=x, \dot{x}_{1}=x_{2}, \ddot{x}_{1}=\dot{x}_{2}$

then equation (12) can be written as

$$
\dot{x}_{2}+p x_{1}=0
$$

The equivalent system is given by

$$
\begin{aligned}
& \dot{x}_{1}=x_{2} \\
& \dot{x}_{2}=-p x_{1}
\end{aligned}
$$

(14) can be reduced in matrix form as

$$
\left(\begin{array}{l}
\dot{x}_{1} \\
\dot{x}_{2}
\end{array}\right)=\left(\begin{array}{cc}
0 & 1 \\
-p & 0
\end{array}\right)\left(\begin{array}{l}
x_{1} \\
x_{2}
\end{array}\right)
$$

(15) can further be written as

$$
\dot{x}=A x
$$

where, $\dot{x}=\left(\begin{array}{l}\dot{x}_{1} \\ \dot{x}_{2}\end{array}\right), x=\left(\begin{array}{l}x_{1} \\ x_{2}\end{array}\right)$ and $A=\left(\begin{array}{cc}0 & 1 \\ -p & 0\end{array}\right)$

For the eigenvalue of $A$ we compute

$$
\begin{gathered}
|A-I \lambda|=0 \\
\left.|A-I \lambda| \Rightarrow \mid \begin{array}{cc}
0 & 1 \\
-p & 0
\end{array}\right)-\lambda\left(\begin{array}{ll}
1 & 0 \\
0 & 1
\end{array}\right) \mid=0 \\
\Rightarrow\left|\left(\begin{array}{cc}
0 & 1 \\
-p & 0
\end{array}\right)-\left(\begin{array}{cc}
\lambda & 0 \\
0 & \lambda
\end{array}\right)\right|=0 \\
\Rightarrow\left|\begin{array}{cc}
-\lambda & 1 \\
-p & -\lambda
\end{array}\right|=0 \\
\lambda^{2}+p=0
\end{gathered}
$$


Evaluating $\lambda$ in (19) we have

$$
\lambda= \pm i p^{\frac{1}{2}}
$$

Since the eigenvalue is in complex form with real part equals to zero, then the equilibrium point is unstable.

\subsection{Convergence of Mathieu Equation}

We consider the Mathieu equation of the form;

$$
\frac{\mathrm{d}^{2} x}{\mathrm{~d} t^{2}}+p x=0
$$

From (21) we assume a power series solution of the form;

$$
x=\sum_{n=0}^{\infty} a_{n} t^{n+k}
$$

Differentiating (21) term by term we have

$$
\begin{gathered}
\dot{x}=\sum_{n=0}^{\infty}(n+k) a_{n} t^{n+k-1} \\
\ddot{x}=\sum_{n=0}^{\infty}(n+k)(n+k-1) a_{n} t^{n+k-2}
\end{gathered}
$$

Substituting (23) and (24) into (21) we have

$$
\sum_{n=0}^{\infty}(n+k)(n+k-1) a_{n} t^{n+k-2}+p \sum_{n=0}^{\infty} a_{n} t^{n+k}=0
$$

The indicial equation is obtained by looking at the coefficient of the lowest power $x^{k-2}$. Since this only occur in the first sum (for $n=0$ ), it must vanish. This reduces (25) to

$$
k(k-1) a_{0} t^{k-2}=0
$$

There are therefore two possible values of the index $k=0, k=1$ and this is quite typical for a second order equation. Changing the dummy index in the first sum by $n \rightarrow n+2$ we have

$$
\sum_{n=-2}^{\infty}(n+k+2)(n+k+1) a_{n+2} t^{n+k}+p \sum_{n=0}^{\infty} a_{n} t^{n+k}=0
$$

All the powers now look alike and we can now compare coefficient to obtain the recurrence relation given by

$$
(n+k+2)(n+k+1) a_{n+2}+p a_{n}=0
$$

which reduces to

$$
a_{n+2}=\frac{-p}{(n+k+2)(n+k+1)} a_{n}
$$

Given the value of $a_{0}$, we can evaluate $a_{2}, a_{4}$, etc. The odd $a_{n}$ are completely independent and as far as getting a solution is concerned, we can put them all to zero. This independent of the odd and even $a_{n}$ is a consequence of the fact that odd and even solution of the differential equation are possible.

In order to generate these odd/even solution, it is easiest to put $a_{1}=0$ in order not to create extra solution by merely using some of the key solution into that of $k=0$. 
The recurrence relation for $k=0$ given by

$$
a_{n+2}=\frac{-p}{(n+2)(n+1)} a_{n}
$$

has the solution

$$
x(t)=a_{0} \cos t+a_{1} \sin t
$$

The recurrence relation for $k=1$ is

$$
a_{n+2}=\frac{-p}{(n+3)(n+2)} a_{n}
$$

with the solution

$$
x(t)=a_{1} \sin t+a_{0} \cos t
$$

Recall that

$$
\begin{aligned}
& \cos t=\sum_{n=0}^{\infty} \frac{(-1) t^{2 n}}{(2 n) !}=1-\frac{t^{2}}{2 !}+\frac{t^{4}}{4 !}-\cdots \\
& \sin t=\sum_{n=0}^{\infty} \frac{(-1)^{n} t^{2 n+1}}{(2 n+1) !}=t-\frac{t^{3}}{3 !}+\frac{t^{5}}{5 !}-\cdots
\end{aligned}
$$

Combining equations (31) and (33) we have

$$
x(t)=a_{0} \cos t+a_{1} \sin t
$$

where $a_{0}$ and $a_{1}$ are arbitrary parameter

Using Ratio Test on $\cos t$, we have

$$
\begin{aligned}
\lim _{n \rightarrow \infty}\left|\frac{a_{n+1}}{a_{n}}\right| & =\lim _{n \rightarrow \infty}\left|\frac{t^{2 n+2}}{(2 n+2) !} \cdot \frac{(2 n) !}{t^{2 n}}\right| \\
& =\lim _{n \rightarrow \infty}\left|\frac{t^{2}}{(2 n+2) !} \cdot \frac{(2 n) !}{(2 n+1)(2 n) !}\right|=\lim _{n \rightarrow \infty} \frac{1}{4 n^{2}-6 n+2}\left|t^{2}\right| \\
& =\lim _{n \rightarrow \infty} \frac{1}{4 n^{2}-6 n+2}\left|t^{2}\right|=0
\end{aligned}
$$

Similarly for $\sin x=\frac{\sum_{n=0}^{\infty}(-1)^{n} t^{2 n+1}}{2 n+1}$ we have

$$
\begin{aligned}
\lim _{n \rightarrow \infty}\left|\frac{a_{n+1}}{a_{n}}\right| & =\lim _{n \rightarrow \infty}\left|\frac{t^{2 n+2}}{(2 n+3) !} \cdot \frac{(2 n+1) !}{t^{2 n-1}}\right| \\
& =\lim _{n \rightarrow \infty}\left|\frac{t^{2}}{(2 n+3)(2 n+2)}\right|=0
\end{aligned}
$$

Thus the interval of convergence is $(\infty,-\infty)$, radius $=$ infinity

Therefore

$$
\begin{aligned}
x(t) & =a_{0} \cos t+a_{1} \sin t \\
& =a_{0} \cdot 0+a_{1} \cdot 0 \\
& =0
\end{aligned}
$$


Since the limit of the odd and even function is equal to zero we conclude that the solution of Mathieu equation converges. Applying the boundary condition we have that

$$
x(0)=x(2 \pi)=a_{1}
$$

\subsection{Numerical Simulation of Forced Mathieu's Equation}

$$
p=0.01
$$

Define a function that determines a vector of derivatives values at any solution point $(t, X)$

$$
D(t, X):=\left[\begin{array}{c}
X_{1} \\
-100 X_{0}-p\left(X_{0}\right)^{2}
\end{array}\right]
$$

Define an additional argument for solving the ODE

$t_{0}:=0 \quad$ Initial value of independent variable

$t_{1}:=10 \quad$ Final value of independent variable

$X_{0}:=\left(\begin{array}{l}0 \\ 1\end{array}\right) \quad$ Vector of initial function values

$N:=1500 \quad$ Numbers of solution values on $\left[t_{0}, t_{1}\right]$

$$
S:=\left(X_{0}, t_{0}, t_{1}, N, D\right)
$$

$t:=S^{\langle 0\rangle} \quad$ Independent variables values

$X_{1}:=S^{\langle 1\rangle} \quad$ First solution function values

$X_{2}:=S^{\langle 2\rangle} \quad$ Second solution function values

\section{Conclusions}

From our result, we observed that the solution existed using the Frobenius method and also periodic. The solution converged at the equilibrium point but unfortunately this convergence did not imply asymptotic stability, and the converse is true. The solution was observed to be unbounded for the given parameters $w$ - $\mathcal{E}$. Since the solution is unbounded, we concluded that the corresponding equilibrium point in $w$ - $\varepsilon$ plane is unstable. This can be seen in Figure 3 where the phase portrait is far away from the equilibrium point. Due to the periodic nature of Mathieu's equation, its application can be seen in propagation of waves in radios and television. MATHCAD software was also used to demonstrate the numerical behavior of the solution of Mathieu's equation. The numerical behavior is explained as follows:

In Figure 1, periodic solution of Mathieu equation was shown by the relationship between the first solution function values and the independent variable values as seen in Table 1 . The variable values were generated using the vector of initial function values $X_{0}$ and the constant $p$. The periodic nature shown is unstable since the trajectory did not start from the equilibrium point. The maximum displacement reached by the trajectory is at \pm 0.1 representing a value along the first solution function values. 
Table 1. The solution matrix table for solution function values.

\begin{tabular}{cccc}
\hline & 0 & 1 & 2 \\
\hline 0 & 0 & 0 & 1 \\
2 & 6.667 & 6.662 & 0.998 \\
3 & 0.013 & 0.013 & 0.991 \\
4 & 0.02 & 0.02 & 0.98 \\
5 & 0.027 & 0.026 & 0.965 \\
6 & 0.033 & 0.033 & 0.945 \\
7 & 0.04 & 0.039 & 0.921 \\
8 & 0.047 & 0.045 & 0.893 \\
9 & 0.053 & 0.051 & 0.861 \\
10 & 0.06 & 0.056 & 0.825 \\
11 & 0.067 & 0.062 & 0.786 \\
12 & 0.073 & 0.067 & 0.743 \\
13 & 0.08 & 0.072 & 0.697 \\
14 & 0.087 & 0.076 & 0.647 \\
15 & 0.093 & 0.08 & 0.595 \\
\hline & 0.1 & 0.084 & $\ldots$ \\
\hline
\end{tabular}

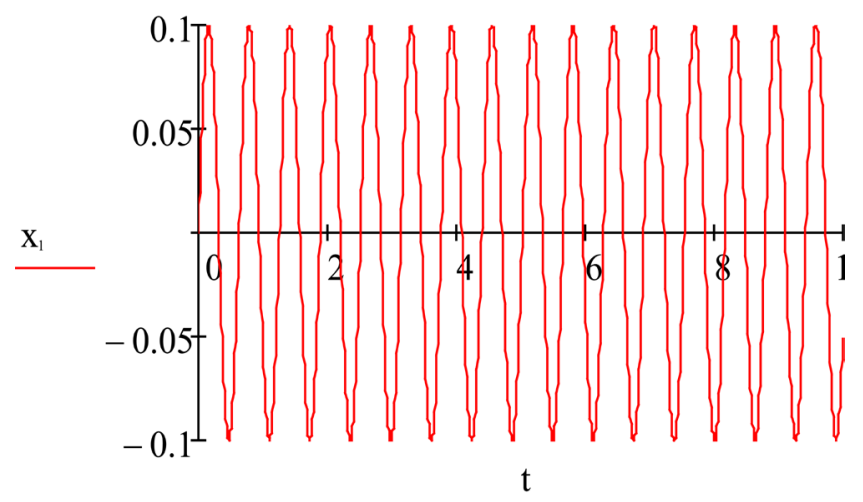

Figure 1. Periodic profile of Mathieu's equation obtained by plotting $X_{1}$ against $t$ for $N=1500$.

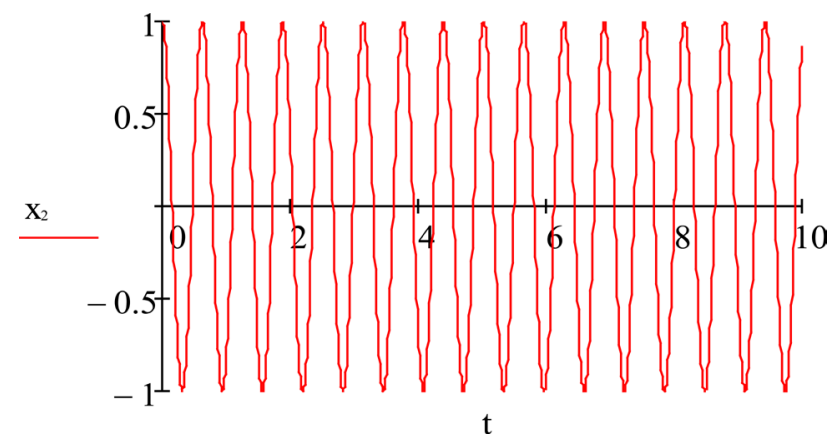

Figure 2. Periodic profile of Mathieu's equation obtained by plotting $X_{2}$ against $t$ for $N=1500$. 




Figure 3. Phase portrait showing instability of solution of Mathieu's equation for $N=1500$.

In Figure 2, the solution was also periodic using the second solution function values and independent variable values. The starting point of the trajectory was far from the origin hence showing instability of the system.

In Figure 3, phase portrait of Mathieu's equation was obtained by relating $X_{2}$ and $X_{1}$. The phase portrait was seen to be far away from the equilibrium point. This shows that the solution of Mathieu's equation is unstable with the maximum displacement coinciding with the maximum displacement of $X_{1}$ in Figure 1. These values represent the maximum interval of stability of the system.

\section{Conflicts of Interest}

The authors declare no conflicts of interest regarding the publication of this paper.

\section{References}

[1] Erwin, K. (2011) Advanced Engineering Mathematics. International Student Version, 10th Edition, John Wiley \& Sons Inc., Hoboken, 180-182. http://www.wiley.com/college/kreyszig

[2] Ruby, L. (1996) Application of Mathieu Equation. American Journal of Physics, 64, 39. https://doi.org/10.1119/1.18290

[3] Chavez-Cerd, S., Padgett, M.J., Allison, I., New, G.H.C., Gutierrez-Vega, J.C., O’Neil, A.T., MacVicar, I. and Courtial, J. (2002) Holographic Generation and Orbital Angular Momentum of High-Order Mathieu Beams. Journal of Optics B: Quantum and Semiclassical Optics, 4, S52-S57.

https://iopscience.iop.org/article/10.1088/1464-4266/4/2/368 https://doi.org/10.1088/1464-4266/4/2/368

[4] Abramowitz, M. and Stegun, I.A. (1964) Handbook of Mathematical Functions. Dover Publications, New York

[5] Cador, C.L. and Ley-koo, E. (2002) Mathieu Function Revisited: Matrix Evaluation and Generating Function. Revista Mexicana De Fisica, 48, 67-75.

[6] Prikhodko, A.A., Nesterov, A.V. and Nesterov, S.V. (2016) Analysis of Mathieu Equation Stable Solution in the First Zone of Stability. Procedia Engineering, 150, 341-346. https://www.elsevier.com/locate/procedia https://doi.org/10.1016/j.proeng.2016.06.715

[7] Rand, R.H. (2016) Lecture Note on Mathieu Equation. Cornell University Italy, 
$1-14$.

http://media.cism.it/courses\%2FA1602\%2Frand\%2Fcourses_A1602_rand_mathieu =CISM.pdf

[8] Bildik, N. and Deniz, S. (2015) On the Asymptotic Stability of Some Particular Differential Equations. International Journal of Applied Physics and Mathematics, 5, 252-258. https://doi.org/10.17706/ijapm.2015.5.4.252-258

[9] Alonso, J.M. (1995) Optimal Interval of Stability of a Forced Oscillator. Proceedings of the American Mathematical Society, 123, 2031-2040.

http://www.ams.org/journal-terms-of-use https://doi.org/10.1090/S0002-9939-1995-1301005-7

[10] Ortega, R. (1992) The First Interval of Stability of a Periodic Equation of Duffing type. Proceeding of American Mathematical Society, 115, 1061-1067.

https://www.ams.org/proc/1992-115-04 https://doi.org/10.1090/S0002-9939-1992-1092925-7

[11] McLachlan, N.W. (1947) Theory and Application of Mathieu Function. Oxford Clarendon Press, Amen House, London, 1-423. https://archive.org/details/in.ernet.dli.2015.215548/page/n45/mode/2up

[12] Polyak, B.T. and Nazin, S.A. (2004) Interval Solution of Interval Algebraic Equations. Journal of Mathematics and Computer in Simulation, 66, 207-217. https://doi.org/10.1016/j.matcom.2003.11.006

[13] Diblik, J., Ruzickova, M. and Suta, Z. (2010) Asymptotical Convergence of the Solutions of a Linear Differential Equation with Delays. Advances in Difference Equations, 2010, Article ID: 749852. https://doi.org/10.1186/1687-1847-2010-749852

[14] Nasabzadeh, H. and Toutounian, F. (2013) Convergent Homotopy Analysis Method for Solving Linear Systems. Advances in Numerical Analysis, 2013, Article ID: 732032. https://doi.org/10.1155/2013/732032

[15] Pipes, L.A. (2004) Matrix Solution of Equation of the Mathieu-Hill Type. Journal of Applied Physics American Institute of Physics, 24, 902. https://doi.org/10.1063/1.1721400

[16] Eze, E.O. (2014) On Application of Lyapunov and Yoshizawa's Theorems on Stability, Asymptotic Stability, Boundedness and Periodicity of Solution of Duffing Equation. ASIAN Journal of Applied Science, 2, 630-635. https://ajouronline.com/index.php/AJAS/article/view/2034

[17] Lazer, A.C. and McKenna, P.J. (1990) On the Existence of Stable Periodic Solutions of Differential Equations of Duffing Type. Proceeding of American Mathematical Society, 110, 125-133. https://www.jstor.org/stable/2048251 https://doi.org/10.2307/2048251

[18] Ogundare, B.S. (2009) Stability and Boundedness Properties of Solution to Certain Fifth Order Nonlinear Differential Equation. Matematicki Vesnik, 4, 257-268.

[19] Edwards, J.T. (2002) Boundedness and Stability of Solution to Difference Equation. Journal of Computational and Applied Mathematics, 140, 275-289. https://doi.org/10.1016/S0377-0427(01)00480-0

[20] Haarsa, P. (2014) The Frobenius Method on a Second Order Homogeneous Linear Ordinary Differential Equation. Advanced Studies in Theoretical Physics, 8, 1145-1148. https://doi.org/10.12988/astp.2014.4798 